\title{
Distribution of human papillomavirus genotypes in invasive squamous carcinoma of the vulva
}

\author{
Bobbie C Sutton ${ }^{1,2}$, Richard A Allen ${ }^{1}$, William E Moore ${ }^{3}$ and S Terence Dunn ${ }^{1}$ \\ ${ }^{1}$ Department of Pathology, The University of Oklahoma Health Sciences Center, Oklahoma City, OK, USA; \\ ${ }^{2}$ Department of Pathology, The South Bend Medical Foundation, Inc., South Bend, IN, USA and ${ }^{3}$ Department \\ of Biostatistics and Epidemiology, Prevention Research Center, The University of Oklahoma Health Sciences \\ Center, Oklahoma City, OK, USA
}

\begin{abstract}
Many studies have established a critical role for human papillomavirus (HPV) in the development of anogenital squamous neoplasia. In this report, we show the distribution of 37 high- and low-risk HPV types in 116 cases of invasive squamous vulvar carcinoma. Sections from paraffin-embedded tissue blocks were dissected as necessary to select areas of invasive carcinoma. Clinical and pathologic variables were analyzed using $t$-tests, univariate odds ratios and logistic regression analysis. Seventy percent of cases were HPV-positive, with an average patient age of 65 years $(n=81)$. HPV-negative cases $(n=35)$ had a higher average age $(70$ years), but these populations were not statistically different $(t=1.65, P=0.10)$. HPV16 was most common $(n=65)$. Other HPV types were less frequent (HPV33, $n=12$; HPV45, $n=4$; HPV52 and 6, each $n=3$; HPV18, 53 and 62, each $n=2)$. Additional HPV types were identified only once. Multiple infections typically included HPV16 (12/14 cases). Tumors showing low-risk HPV (11 cases) and low-risk HPV only (three cases) were uncommon. Regional node metastasis was documented in 29 of 116 tumors, and 8/9 HPV-positive nodes contained HPV types identical to the primary tumor. Of tumor types, warty carcinoma was most strongly associated with highrisk HPV (odds ratio 4.34, 95\% confidence interval 1.32-18.45), particularly high-risk HPVs other than type 16 (odds ratio 9.04, 95\% confidence interval 1.60-54.00). Tumors associated with any HPV type (odds ratio 0.40, $95 \%$ confidence interval $0.14-1.17$ ), any high-risk type (odds ratio $0.36,95 \%$ confidence interval $0.12-1.08$ ), or type 16 alone (odds ratio $0.34,95 \%$ confidence interval $0.11-1.12$ ) were less likely to metastasize than HPV-negative tumors. Correcting for possible confounding variables, such as patient age and tumor histology, linear logistic regression analysis confirmed this association (high-risk HPV odds ratio 0.28 , $95 \%$ confidence interval 0.09-0.89).

Modern Pathology (2008) 21, 345-354; doi:10.1038/modpathol.3801010; published online 11 January 2008
\end{abstract}

Keywords: vulvar cancer; linear array HPV test; human papilloma virus; genotyping; lymph node metastasis

According to American Cancer Society statistics, carcinoma of the vulva accounts for about $4 \%$ of all malignancies in the female reproductive tract. In the United States, there will be an estimated 3490 new cases identified and 880 deaths due to the disease during 2007. ${ }^{1}$ This relatively uncommon malignancy is predominantly seen in older women in the sixth to ninth decades of life; however, women aged 35 and under may also be affected, and recent data suggest that the incidence of vulvar neoplasia

Correspondence: Dr S Terence Dunn, PhD, Department of Pathology, University of Oklahoma Health Sciences Center, 940 Stanton L Young Blvd., Biomedical Sciences Buidling 451, Oklahoma City, Oklahoma 73104, USA.

E-mail: terry-dunn@ouhsc.edu

Received 07 August 2007; revised 24 October 2007; accepted 31 October 2007; published online 11 January 2008 in younger women is increasing. ${ }^{2-4}$ The standard treatment for vulvar cancer remains surgical, but there has been a trend toward more limited resection due to a number of factors, including the significant morbidity associated with radical vulvectomy, the application of other treatment modalities, and the substantial psychosexual adjustment often required after genital surgery. ${ }^{5}$ The 5 -year survival ranges from $29-98 \%$ for the malignancy depending on stage, but individual prognoses are affected by multiple factors, principally the type of tumor and the patient's age, and general health. ${ }^{1,6}$

By far the most common type of vulvar tumor is squamous cell carcinoma, which accounts for almost $90 \%$ of cases. Like squamous carcinoma found at other anogenital sites such as the cervix, there is accumulating evidence that human papillomavirus 
(HPV) infection is associated with the development of vulvar neoplasia, both in-situ and invasive; although, patients with a history of sexually transmitted disease or smoking also tend to be more prone to the malignancy. ${ }^{7}$ In excess of $100 \mathrm{HPV}$ genotypes are recognized based on specific DNA sequence variations and $30-40$ are associated with anogenital lesions. However, the oncogenic capacity of individual HPV types differs considerably. ${ }^{8,9}$ Accordingly, anogenital HPVs have been divided into high- and low-risk types based on the related disease risk. Most cases of cervical precancerous and cancerous lesions are associated with persistent infection involving one or more of approximately 15 high-risk genotypes. Four genotypes in particular, HPV types 16, 18, 31, and 45, account for the great majority of cervical cancer worldwide although regionally a substantial role is also contributed by HPV types 52 and 58 in Asia and HPV33 in Europe. ${ }^{10,11}$

Unlike cervical cancer where the association with HPV is virtually absolute, HPV DNA has been detected in cases of invasive squamous vulvar carcinoma with a broad reported range $(0-85 \%)$ of positive results. ${ }^{2,7,12-33}$ Some of the variation in HPV prevalence between studies may be due to age or variability in patient populations assessed. Multiple authors have suggested that vulvar cancer comprises two separate diseases, the first type occurring with high prevalence among young women and developing from vulvar intraepithelial neoplasia caused by HPV, and a second type afflicting older women and developing from non-neoplastic epithelial disorders such as lichen sclerosus or chronic inflammation..$^{2-4,12,13,15,18,24,25}$ However, these studies of HPV prevalence in invasive squamous vulvar carcinoma also utilized different methods of detection, including in-situ, ${ }^{13-18}$ dot blot, ${ }^{20,21}$ and Southern blot $^{20}$ hybridization, immunohistochemistry, ${ }^{15}$ and PCR-based assays, ,2,12,18,19,22-24,26-31,33-36 each with different analytic sensitivities. Moreover, the particular HPV genotype(s) targeted in previous work is inconsistent between studies; many early reports documented only HPV16, 18, 33, and/or $6 / 11,{ }^{18,20,21,26,28-30}$ while later work targeted more than 20 different HPV types. ${ }^{12,22}$ Also, some studies tested only a few cases of invasive squamous vulvar carcinoma, which limits the predictive value of the resulting data. ${ }^{14,17,21,23}$ Despite these shortcomings, this body of work indicates that HPV16 is the most common genotype present in invasive squamous vulvar carcinoma. ${ }^{2,7,16-19,22,24,26-31}$ The prevalence of other high- and low-risk HPV types in invasive squamous vulvar carcinoma, however, remains less certain.

The present study describes the distribution of 37 anogenital HPV types in 116 cases of invasive squamous vulvar carcinoma using a sensitive PCR-based, reverse blot hybridization detection method. The resulting data are correlated with other clinicopathologic prognostic variables.

\section{Materials and methods}

\section{Tumor Cohort}

With local Institutional Review Board approval, 116 cases of invasive squamous cell carcinoma of the vulva were identified in the surgical pathology records of the OU Medical Center, Oklahoma City, dating from 1987 to 2007. Computerized record searching was not available for the oldest cases; therefore, the 116 cases represent a subset of all vulvar carcinomas treated at this institution during this time period.

Cases were included in the study only when appropriate pathology materials were available: standard neutral-buffered formalin-fixed tissue embedded in paraffin block(s) demonstrating invasive tumor, with corresponding surgical pathology reports. However, not all of the original slides and blocks were available for review in every case. Cases were reviewed by a surgical pathologist (BCS) to confirm the diagnosis of record. All tumors in this cohort were invasive squamous cell carcinomas. These cases were further subdivided into specific morphologic variants of squamous cell carcinoma known to occur in the vulva. Basaloid carcinoma and warty carcinoma were the only variant squamous cell carcinoma types encountered in this data set and have been previously described. ${ }^{18,32}$ Briefly, basaloid carcinoma consists of variably sized cords, sheets, and nests of immature-appearing tumor cells resembling basal squamous cells with little or no maturation, abundant mitotic activity, and a densely hyalinized supporting stroma (Figure 1a). Warty carcinoma often has a papillomatous tumor surface, and is composed of neoplastic cellular nests, sometimes with centralized keratin whorls, and individual cell keratinization. Warty carcinoma typically shows nuclear features characteristic of HPV infection: enlarged, pleomorphic nuclei with nuclear membrane irregularities, and perinuclear halos (Figure 1b). Any squamous cell carcinoma that did not meet the morphologic criteria of these two specific categories was designated squamous cell carcinoma, not otherwise specified (Figure 1c).

Complete clinicopathologic staging information fulfilling American Joint Committee on Cancer staging criteria (TNM classification) for vulvar carcinoma was not available in all cases. We only analyzed information that was routinely available in archival surgical pathology reports, such as tumor size, depth of tumor invasion, and presence/absence of lymph node metastasis.

\section{Tissue Processing and DNA Extraction}

Hematoxylin and eosin (H\&E) stained sections from each block were used to identify areas of squamous cell carcinoma for DNA extraction. Unstained, 5- $\mu \mathrm{m}$ thick tissue sections were dissected manually when necessary to ensure selection of the invasive tumor 

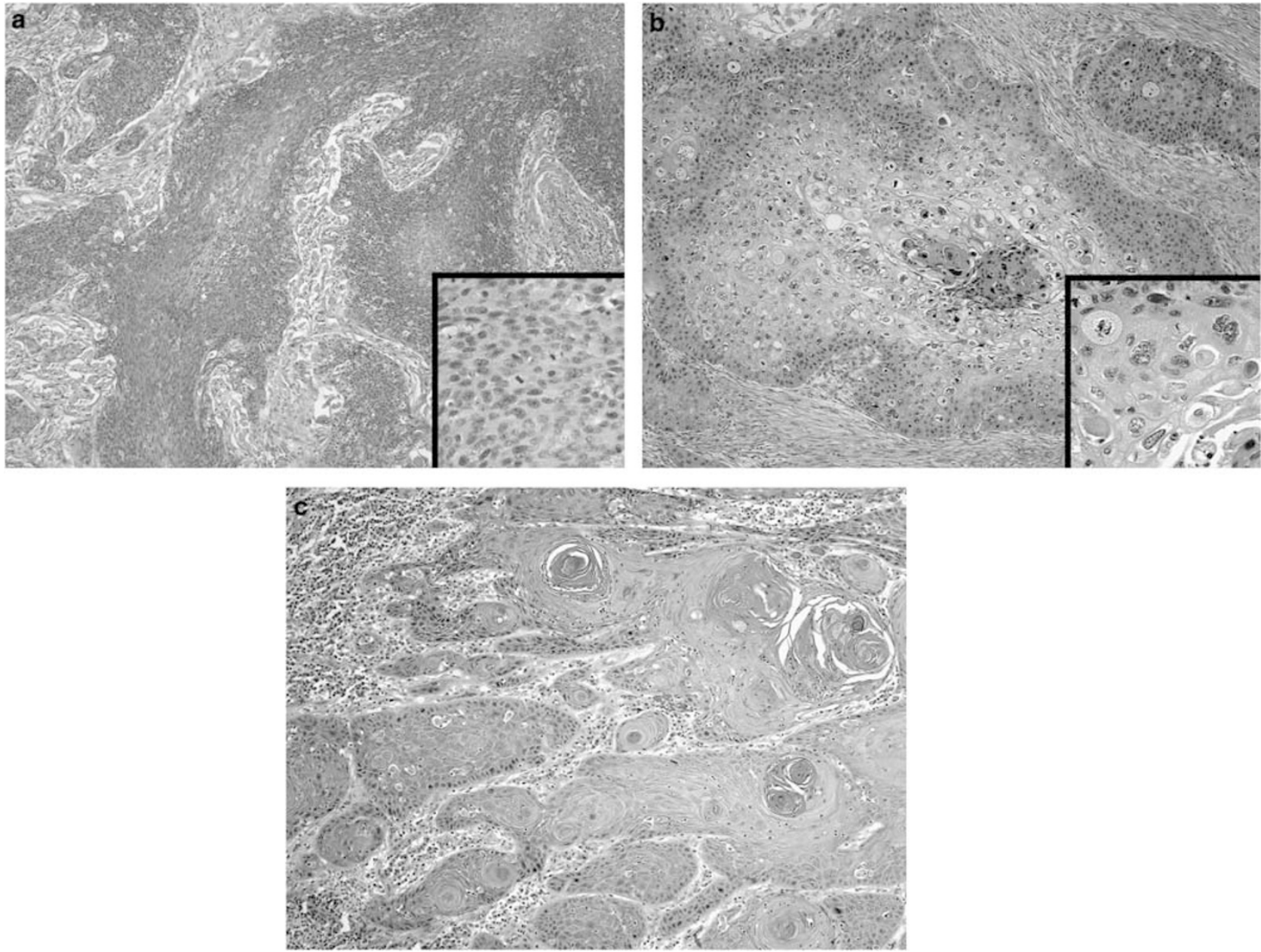

Figure 1 Representative H\&E histologies of invasive squamous vulvar carcinoma. (a) Basaloid carcinoma composed of cords and irregularly shaped aggregates of closely packed neoplastic cells showing little evidence of squamous maturation, which are set within a fibrous stroma. Inset shows higher magnification view of the relatively uniform, oval nuclei, and mitotic activity. (b) Warty carcinoma island composed of abnormal squamous cells with abundant pale cytoplasm and central parakeratosis. Inset highlights an area of pleomorphic nuclei with features associated with HPV infection; irregular nuclear contours, perinuclear clear zones or halos, and individually dyskeratotic cells. (c) Conventional invasive squamous cell carcinoma, not otherwise specified, with an infiltrative margin and keratinous whorls. (Magnification $\times 100$ with $\times 400$ insets.)

component. Any vulvar intraepithelial neoplasia noted adjacent to an invasive tumor was avoided when selecting an area for DNA extraction. Techniques to prevent carry over contamination, as previously described, ${ }^{22,33,37}$ were routinely applied throughout tissue handling. Whole or manually dissected tissue sections were deparaffinized with xylene (three 10-min changes), rinsed with absolute ethanol (three 5-min changes), then digested with proteinase $\mathrm{K}$ in ATL tissue lysis buffer (DNeasy Tissue kit; Qiagen, Valencia, CA, USA) at $55^{\circ} \mathrm{C}$ for at least $24 \mathrm{~h}$. Digests were subsequently processed for DNA extraction using the DNeasy Tissue Kit according to the manufacturer's instructions. Whenever possible, a second H\&E-stained slide was prepared subsequent to cutting sections for DNA extraction to reconfirm the histologic pattern of the tumor. Extracted DNA was quantified by spectrophotometry for each case; concentrations ranged from 4 to $127 \mathrm{ng} / \mu \mathrm{l}$. For HPV-positive tumors with regional lymph node involvement, DNA from nodes containing metastatic squamous carcinoma was also extracted in a similar fashion.

\section{HPV Genotyping}

Isolated DNA was subjected to PCR and HPV detection using the Roche LINEAR ARRAY ${ }^{\circledR}$ HPV Genotyping Test (Roche Diagnostics, Branchburg, NJ, USA), as previously described. ${ }^{38}$ This test was designed to allow for the simultaneous identification of 37 carcinogenic and non-carcinogenic anogenital HPV types $(6,11,16,18,26,31,33,35,39$, $40,42,45,51,52,53,54,55,56,58,59,61,62,64$, $66,67,68,69,70,71,72,73,81,82,83,84$, IS39, and CP6108) from liquid-based cervical cell preparations (PreservCyt ${ }^{-}$Solution, Cytyc Corp., Boxborough, MA, USA). The performance of the Linear Array HPV Test using DNA extracted from 
paraffin-embedded tissue has not been evaluated by the manufacturer but the assay has demonstrated robust performance (as demonstrated by high reproducibility of results from the same samples) in our laboratory using this tissue type and in the hands of others using DNA extracted from frozen tissue. $^{39}$ The assay uses a pool of biotinylated primers that targets the polymorphic L1 gene for PCR amplification. Biotinylated amplicons (approximately $450 \mathrm{bp}$ ) are then hybridized to 37 immobilized capture probes representing different HPV genotypes. Simultaneous amplification of a $268 \mathrm{bp}$ fragment of the human $\beta$-globin and subsequent hybridization of these biotinylated amplicons to low- and high-sensitivity $\beta$-globin capture probes serves as an internal control to monitor for optimal amplification of each sample. Any specimen that failed to yield a positive reaction with the highsensitivity $\beta$-globin probe was considered a test failure and results were not included in the study. When the high-concentration $\beta$-globin band was weak or there was a very weak HPV-specific band present, DNA was re-extracted from the same block and the test was repeated, or alternatively the same DNA was reanalyzed. A negative (no DNA template) control was included with every PCR to monitor for sample contamination and never showed any reaction. For quality assurance purposes, the same tissue block was extracted more than one time or the same DNA from some cases was amplified on more than one occasion, and these samples were re-tested in the Linear Array HPV assay.

\section{Statistical Analysis}

Categorical variables such as tumor histology, HPV genotypes, and nodal metastasis status were summarized by frequencies and percentages. Continuous variables such as patient age at diagnosis, tumor size, and depth of invasion were summarized by mean, range, and s.d. $\chi^{2}$ tests and odds ratios with exact confidence intervals were used to compare categorical variables among vulvar carcinomas with or without high- and low-risk HPV genotypes, tumor histology, and presence or absence of nodal metastasis. Independent two-tailed Student's $t$-tests were used for comparisons of age, tumor size, and depth of invasion by HPV status. Multivariate linear logistic regression analysis was used to assess the relationship of independent variables to nodal metastasis. Statistical analysis was performed using NCSS 2007 (NCSS, Kaysville, UT, USA) and Stata Statistical Software: Release 9.0 (Stata Corp., College Station, TX, USA).

\section{Results}

Eighty-one (70\%) of the 116 invasive squamous carcinomas of the vulva analyzed were positive for one or more HPV genotypes using the Linear Array
Table 1 Prevalence of HPV genotypes in invasive squamous vulvar carcinoma cases

\begin{tabular}{lcc}
\hline & $\begin{array}{c}\text { Cases } \\
(\mathrm{n})\end{array}$ & $\begin{array}{c}\text { Frequency } \\
(\%)\end{array}$ \\
\hline HPV-positive cases & 81 & $69.8^{\mathrm{a}}$ \\
& & \\
All cases with & & \\
High-risk HPV(s) & 78 & 96.3 \\
HPV16 & 65 & 80.2 \\
HPV 33 & 12 & 14.8 \\
HPV 45 & 4 & 4.9 \\
HPV 52 & 3 & 3.7 \\
HPV 18 & 2 & 2.5 \\
Low-risk HPV & 11 & 13.6 \\
Multiple HPV types & 14 & 17.3 \\
Multiple HPV types, including HPV 16 & 12 & 14.8 \\
Cases only with & & \\
High-risk HPV(s) & 70 & 86.4 \\
Low-risk HPV & 3 & 3.7 \\
Single HPV type & 68 & 83.9 \\
\hline
\end{tabular}

${ }^{\mathrm{a}}$ Expressed as percentage of total cases $(n=116)$. All other frequencies are expressed as a percentage of HPV-positive cases $(n=81)$.

${ }^{b}$ High-risk HPV cases reflect each time that the genotype was detected. Multiple infections may therefore be counted more than once.

HPV Test (Table 1). HPV16 was the most commonly detected genotype, occurring in $80 \%$ of HPVpositive cases, followed by HPV33 (15\%). Other high-risk genotypes detected included HPV45, 52, and 18 which were each present in less than $5 \%$ of HPV-positive cases. Low-risk HPV genotypes were identified in 11 cases (14\%). Only three cases $(4 \%)$ revealed solely low-risk HPV types, and included one case each of HPV6, 26, and 61. Infections with a single HPV type were most common (83\% of HPVpositive cases), with HPV16 comprising the most frequent type of single infection $(79 \%$ of HPVpositive cases). Of 14 cases infected with multiple HPV genotypes, 12 (86\%) also harbored HPV16 (Table 2). A maximum of three different HPV genotypes were identified in two separate cases.

The average age of all patients in the study was 66 years. Patients with HPV-positive tumors $(n=81)$ had an average age of 65 years while HPV-negative cases $(n=35)$ had a slightly higher average age (70 years); however, these two populations were not statistically different $(t=1.65, P=0.10)$. Seventy-six of the 116 patients underwent primary vulvectomy (often radical or modified radical), with regional inguinofemoral lymph node sampling. Twenty-two surgical procedures were vulvar excisions of variable extent without lymphadenectomy. Four patients with advanced disease underwent excision of the vulva as well as other adjacent organs such as urethra, vagina, and/or rectum. In 14 patients, the primary diagnostic vulvar biopsy was used for study.

The cases were divided morphologically into 71 conventional squamous cell carcinomas, not 
otherwise specified, 35 warty carcinomas, and 10 basaloid carcinomas. Representative examples of each of these histological categories are shown in Figure 1. Of the $81 \mathrm{HPV}$-positive tumors, 44 (54\%) were classified as squamous cell carcinoma, not otherwise specified, 30 (37\%) were warty carcinoma and seven were basaloid carcinoma ( $9 \%)$. Of these tumor types, warty carcinoma was HPV-positive in $86 \%(30 / 35)$ of cases. HPV was also identified in $70 \%$ of basaloid carcinomas (7/10 cases), and in $62 \%(44 / 71)$ of squamous cell carcinoma, not otherwise specified, cases. Compared to the other tumor variants (squamous cell carcinoma, not otherwise specified, and basaloid carcinomas combined), warty carcinoma was most highly associated with high-risk HPV infection (odds ratio 4.34, 95\% confidence interval 1.31-18.45), particularly highrisk HPVs other than type 16 (odds ratio 9.04, 95\% confidence interval 1.60-54.00).

Of 82 cases where regional lymph node status was documented, 29 (35\%) were positive for metastatic squamous carcinoma. Of the 29 cases, 11 had an HPV-positive primary tumor and lymph node tissue

Table 2 Distribution of HPV genotypes in invasive squamous vulvar carcinoma cases

\begin{tabular}{lcccc}
\hline \multicolumn{2}{c}{$\begin{array}{c}\text { Single genotype } \\
\text { HPV infection }\end{array}$} & & \multicolumn{2}{c}{$\begin{array}{c}\text { Multiple genotype } \\
\text { HPV infection }\end{array}$} \\
\cline { 1 - 1 } \cline { 4 - 4 } HPV type & Cases $(\mathrm{n}=67)$ & & HPV types & Cases $(\mathrm{n}=14)$ \\
\hline 16 & 53 & & 16 and 33 & 5 \\
33 & 5 & & 16 and 6 & 2 \\
18 & 2 & & 16 and 45 & 1 \\
45 & 2 & & 16 and 53 & 1 \\
52 & 2 & & 16 and 62 & 1 \\
6 & 1 & & 33 and 67 & 1 \\
26 & 1 & & 16,33 , and 53 & 1 \\
61 & 1 & 45,52 , and 62 & 1 \\
& &
\end{tabular}

blocks that were available for study. Of these 11 cases, two were HPV-negative in the lymph node tissue. Eight of the remaining nodes demonstrated HPV genotypes identical to the primary tumor (HPV16, $n=5$; and one case each of HPV types 18, 26, and 52). In one node-positive case, HPV types 16 and 33 were detected in the node, while the primary tumor demonstrated only HPV33.

Tumors associated with any HPV type (odds ratio $0.40,95 \%$ confidence interval $0.14-1.17$ ), any highrisk type (odds ratio $0.36,95 \%$ confidence interval $0.12-1.07$ ), or type 16 alone (odds ratio $0.34,95 \%$ confidence interval $0.10-1.12$ ) were less likely to metastasize than HPV-negative tumors. Correcting for possible confounding variables, such as patient age and tumor histology, logistic regression analysis confirmed this association (high-risk HPV odds ratio $0.28,95 \%$ confidence interval 0.09-0.89). Other tumor morphologic parameters, such as measured tumor size and invasive tumor depth, did not correlate with metastatic disease or HPV status (data not shown). However, overall depth of invasion was significantly less in warty carcinoma (mean = $0.49 \mathrm{~cm} \pm 0.32 \mathrm{s.d}$.) than that of squamous cell carcinoma, not otherwise specified (mean $=0.78 \mathrm{~cm}$ \pm 0.69 s.d.; $t=2.77, P=0.007$ ) (Table 3 ).

DNA was extracted from a total of 133 tissue blocks (primary tumor and metastatic lymph nodes) representing 121 cases of invasive squamous vulvar carcinoma that met study criteria. Assay failure, possibly due to low DNA concentration, degraded DNA, or the presence of PCR inhibitors, occurred repeatedly with samples extracted from six blocks (5\%), representing five cases. Repeat analysis of failed specimens rarely yielded additional information. Repeat analysis of the same tissue block or the same DNA as part of assay quality assurance occasionally produced discordant results, typically when a weak hybridization band disappeared on the repeat run. This phenomenon has been previously reported for the Linear Array HPV Test. ${ }^{38}$

Table 3 Histological features of invasive squamous vulvar carcinoma cases

\begin{tabular}{|c|c|c|c|c|c|c|}
\hline \multirow[t]{2}{*}{ Variable } & \multicolumn{2}{|c|}{ Squamous cell carcinoma } & \multicolumn{2}{|c|}{ Warty } & \multicolumn{2}{|c|}{ Basaloid } \\
\hline & $\begin{array}{l}\text { Mean } \\
\text { Median }\end{array}$ & s.d. & $\begin{array}{l}\text { Mean } \\
\text { Median }\end{array}$ & s.d. & $\begin{array}{l}\text { Mean } \\
\text { Median }\end{array}$ & s.d. \\
\hline $\begin{array}{l}\text { Tumor size }(\mathrm{cm})^{\mathrm{a}} \\
\text { Kruskal-Wallis ANOVA: } \chi^{2} 0.5\end{array}$ & $\begin{array}{l}2.55 \\
2.5 \\
(n=60)^{\mathrm{b}} \\
76\end{array}$ & 1.58 & $\begin{array}{c}2.81 \\
2.5 \\
(n=27)\end{array}$ & 2.81 & $\begin{array}{c}1.88 \\
1.75 \\
(n=6)\end{array}$ & 1.35 \\
\hline $\begin{array}{l}\text { Depth of invasive tumor }(\mathrm{cm})^{\mathrm{a}} \\
\text { Kruskal-Wallis ANOVA: } \chi^{2} 3.5\end{array}$ & $\begin{array}{c}0.78 \\
0.54 \\
(n=60)\end{array}$ & 0.69 & $\begin{array}{c}0.49 \\
0.49 \\
(n=31)\end{array}$ & 0.32 & $\begin{array}{c}0.62 \\
0.60 \\
(n=7)\end{array}$ & 0.34 \\
\hline
\end{tabular}

${ }^{\mathrm{a}}$ Tumor dimensions based on pathology report and/or the tumor block examined, whichever value greatest.

${ }^{\mathrm{b}}$ Data not available on all specimens due to omission of measurements in pathology reports, or when biopsies studied. 


\section{Discussion}

High-risk, potentially tumorigenic HPV types were frequently identified in invasive squamous vulvar carcinoma in this study, and by far the most common type was HPV16, a finding that agrees with the work of others. ${ }^{2,7,16-19,22,24,26-31}$ The next most frequently identified high-risk type was HPV33, which has also been previously documented in invasive squamous vulvar carcinoma. ${ }^{24,27,29,34,35}$ HPV18, a high-risk genotype that has been typically searched for in invasive squamous vulvar carcinoma by earlier investigators (Table 4), was identified infrequently in the current study ( $3 \%$ of HPV-positive cases). While a previous investigation $^{36}$ reported HPV18 to be commonly associated with invasive squamous vulvar carcinoma (12 of 15 cases), other authors and data from the present study show that HPV18 is detectable in one third or less of total HPV-positive invasive squamous vulvar carcinoma cases ${ }^{2,18,19,21,22,26,27,29,31,34}$ or is absent. ${ }^{16,17,30,35,40}$ The significance of these differences is uncertain, but is likely related to methodologies employed and possible variations in HPV prevalence within the individual populations tested. Other high-risk HPV types identified in the current study included HPV45 (5\% of HPV-positive cases) and 52 (4\% of HPV-positive cases). HPV45 has been infrequently reported in association with invasive squamous vulvar carcinoma. ${ }^{19,24,31}$ The first described case of an HPV52-positive invasive squamous vulvar carcinoma occurred in an 18-year-old female, who developed invasive disease in less than 3 years. ${ }^{42}$ Interestingly, one of three HPV52-positive invasive squamous vulvar carcinoma cases in the current study demonstrated HPV52 in a regional lymph node containing metastatic tumor. These observations, while anecdotal, suggest the possibility of potent oncogenicity for HPV52 in invasive squamous vulvar carcinoma.

It should be noted that high-risk HPVs may also be associated with high-grade vulvar intraepithelial neoplasia, with HPV16 being found in greater than $90 \%$ of cases. ${ }^{37} \mathrm{HPV}$ types $18,31,33,35,51,52$, and 68 have also been infrequently identified in vulvar intraepithelial neoplasia $3 .^{27,37}$ While care was exercised in selecting areas of invasive carcinoma for study which did not have histologic evidence of vulvar intraepithelial neoplasia, we acknowledge that our manual tissue microdissection technique could have had microscopic vulvar intraepithelial neoplasia contamination in some cases, and thus falsely increase the detection of some HPV genotypes. However, overall our results of high-risk HPV prevalence in invasive squamous vulvar carcinoma correlate with previous work. Review of recent literature where highly sensitive PCR-based techniques were applied to the detection of multiple HPV types in larger invasive squamous vulvar carcinoma case series identified a broad reported range of HPVpositive cases, from 0 to $85 \%$ (Table 4). Notably, several of these studies, including the current data, show a 50-70\% HPV-positive rate for invasive squamous vulvar carcinoma. Detection of multiple HPV types in a single tumor, particularly HPV16 combined with another type or types, has also been regularly identified by multiple groups and is noted in the current report (Tables 2 and 4).

Multiple attempts have been made to detect low-risk HPV types 6 and 11 in invasive squamous vulvar carcinoma, with variable results (Table 4). ${ }^{7,12,14-17,19-22,27,31}$ In this report, low-risk HPV types in general were infrequently encountered, and only three invasive squamous vulvar carcinoma cases were identified that contained only low-risk HPV genotypes, correlating with previous work. $^{7,19,21,22,27,31}$ Interestingly, one case where lowrisk HPV26 was identified in a primary warty carcinoma also demonstrated HPV26 in a lymph node which contained metastatic squamous carcinoma. Also, a second patient with only low-risk HPV6 detected in the primary tumor went on to develop metastatic disease in an inguinal node. However, the metastatic squamous cell carcinoma was identified by fine needle aspiration cytology rather than by surgical pathology, and determination of HPV status in the metastatic deposit was not possible for this case.

Recent literature suggests that invasive squamous vulvar carcinoma may be divided into two general categories based on a variety of epidemiologic and clinicopathologic factors. ${ }^{24,12,13,18,24,25}$ Classical HPV-associated squamous carcinoma variants, such as basaloid carcinoma and warty carcinoma, tend to occur more frequently in younger women, contain high-risk HPV types, often have adjacent in situ squamous neoplasia with basaloid or warty features, ${ }^{2,4,18,25,32,37}$ may be multifocal or multicentric, ${ }^{43,44}$ and can involve other anogenital sites. ${ }^{4,28,34}$ In accordance with this previous data, warty carcinoma showed the strongest association with HPV infection in the current study, particularly high-risk HPV. In contrast, squamous cell carcinoma, not otherwise specified, is generally believed to be less likely HPV-related, ${ }^{12,18,32}$ often does not have associated vulvar intraepithelial neoplasia or has simplex type, differentiated, non-HPV associated vulvar intraepithelial neoplasia, ${ }^{12,30,35,45}$ and occurs in older women in their seventh to ninth decades. The reported difference in average, ${ }^{23,30,44}$ or median ${ }^{35,46}$ patient age between HPV-positive and -negative invasive squamous vulvar carcinomas ranges from 12 to more than 20 years in some studies. However, our set of 116 cases demonstrated an average age of patients with HPV-positive invasive squamous vulvar carcinoma that was only slightly lower (65 \pm 16 years) than that of patients with HPV-negative invasive squamous vulvar carcinoma (70 \pm 15 years), and this difference was not statistically significant. Similar findings were noted in a recent study of 48 invasive squamous vulvar carcinoma cases by Hampl et $a l^{27}$ who reported an average age of 
Table 4 Prevalence of HPV as detected by PCR in larger published invasive squamous vulvar carcinoma case series

\begin{tabular}{|c|c|c|c|c|c|c|c|c|c|c|}
\hline Ref. & Description/technique & Cases (n) & $\begin{array}{l}H P V- \\
\text { positive } \\
\text { rate }\end{array}$ & $H P V 16^{\mathrm{a}}$ & $H P V 18^{\mathrm{a}}$ & $H P V 33^{\mathrm{a}}$ & $H P V 45^{\mathrm{a}}$ & $\begin{array}{l}H P V 6 \text { and } \\
\quad \text { or } 11^{\mathrm{a}}\end{array}$ & Multiple types ${ }^{\mathrm{a}}$ & $\begin{array}{l}\text { Unclassified } \\
\text { types }^{\mathrm{a}}\end{array}$ \\
\hline 40 & $\begin{array}{l}\text { PCR with Southern blot for HPV types } \\
6,11,16,18,31,33,35,51 \text {, and } \\
\text { unclassified }\end{array}$ & 23 & $30 \%$ & 4 & 0 & 0 & $N A^{b}$ & 0 & 0 & 3 \\
\hline 18 & $\begin{array}{l}\text { PCR with Southern blot for HPV types } \\
6,11,16 \text {, and } 18\end{array}$ & $22^{\mathrm{c}}$ & $54 \%$ & 10 & 2 & NA & NA & 0 & $\begin{array}{l}\text { HPV16 and } 18, \\
\text { one case }\end{array}$ & NA \\
\hline 34 & $\begin{array}{l}\text { PCR with dot blot for HPV types } 6 / 11 \text {, } \\
16,18 \text {, and } 33^{\mathrm{d}}\end{array}$ & 19 & $31 \%$ & 13 & 3 & 3 & NA & 0 & 0 & NA \\
\hline 35 & $\begin{array}{l}\text { PCR with dot blot for HPV types } 6 / 11 \text {, } \\
16,18 \text {, and } 33\end{array}$ & 78 & $31 \%$ & 21 & 0 & 2 & NA & 0 & $\begin{array}{l}\text { HPV16 and } 33 \\
\text { one case }\end{array}$ & NA \\
\hline 41 & $\begin{array}{l}\text { PCR with Southern blot for HPV types } \\
6 / 11 / 13 / 16 / 18 / 30 / 31 / 32 / 33 / 45 / 51\end{array}$ & 60 & $32 \%$ & NA & NA & NA & NA & NA & NA & NA \\
\hline 22 & $\begin{array}{l}\text { PCR with Southern blot for HPV types } \\
6,16,18 \text {, and unclassified }\end{array}$ & 55 & $60 \%$ & 27 & 3 & NA & NA & 1 & 0 & 2 \\
\hline 29 & $\begin{array}{l}\text { PCR with gel electrophoresis for HPV } \\
\text { types } 6,11,16,18,33 \text {, and unclassified }\end{array}$ & 74 & $36 \%$ & 19 & 9 & 1 & NA & 0 & $\begin{array}{l}\text { HPV16 and 18, two } \\
\text { cases; HPV16 and 33, } \\
\text { one case }\end{array}$ & 1 \\
\hline 7 & $\begin{array}{l}\text { PCR with Southern blot for HPV types } \\
6 / 11,16,18 / 45,31 / 33 / 52 \text {, and } \\
\text { unclassified }\end{array}$ & 55 & $51 \%$ & 24 & 1 & 2 & 1 & 1 & $\begin{array}{c}\text { HPV16 and one } \\
\text { unclassified, } 21 \text { cases; } \\
\text { HPV16 and two } \\
\text { unclassified, two } \\
\text { cases; HPV6/11 and } \\
\text { one unclassified, } \\
\text { two cases }\end{array}$ & 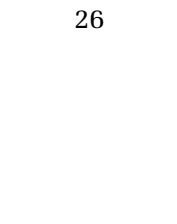 \\
\hline 24 & PCR with sequence analysis & 66 & $20 \%$ & 11 & 0 & 1 & 1 & 0 & 0 & 0 \\
\hline 31 & $\begin{array}{l}\text { PCR with dot blot for HPV types } 16,18 \text {, } \\
45,6 / 11,31 / 33 / 34 / 35 / 39,40 / 42 / 43 / 44 / \\
45, \text { and } 51 / 52 / 54 / 56 / 58\end{array}$ & 158 & $24 \%$ & 21 & 10 & 0 & 1 & 1 & $\begin{array}{l}\text { HPV16 and 18, four } \\
\text { cases; HPV6/11 and } \\
16 \text {, one case }\end{array}$ & NA \\
\hline 2 & $\begin{array}{l}\text { PCR with gel electrophoresis for HPV } \\
\text { types } 16,18 \text {, and unclassified }\end{array}$ & 20 & $85 \%$ & 9 & 4 & NA & NA & NA & HPV16 \& 18, two cases & 2 \\
\hline 12 & $\begin{array}{l}\text { PCR with reverse hybridization line } \\
\text { probe assay (LiPA) for HPV types } 6,11 \text {, } \\
16,18,31,33,34,35,39,40,42,43,44, \\
45,51,52,53,54,56,58,59,66,68,70, \\
\text { and } 74\end{array}$ & 16 & $0 \%$ & 0 & 0 & 0 & 0 & 0 & 0 & NA \\
\hline 27 & PCR with sequence analysis & 48 & $60 \%$ & 19 & 1 & 4 & 0 & NA & 4 & 5 \\
\hline $\begin{array}{l}\text { Current } \\
\text { study }\end{array}$ & $\begin{array}{l}\text { PCR with Linear Array HPV for HPV } \\
\text { types } 6,11,16,18,26,31,33,35,39 \text {, } \\
40,42,45,51,52,53,54,55,56,58,59 \\
61,62,64,66,67,68,69,70,71,72,73 \text {, } \\
81,82,83,84, \text { IS39, and CP6108 }\end{array}$ & 116 & $70 \%$ & 65 & 2 & 12 & 4 & 3 & 14 & 0 \\
\hline
\end{tabular}

${ }^{\mathrm{a}}$ Values for the HPV-positive subcategories do not necessarily sum to the totals because of multiple types identified in individual samples.

${ }^{\mathrm{b}} \mathrm{NA}, \mathrm{HPV}$ genotype not specifically assessed in study.

${ }^{\mathrm{C}}$ Out of 30 reported invasive squamous vulvar carcinoma cases, eight were negative for both HPV and $\beta$-globin control, for a total of 22 cases.

${ }^{\mathrm{d}}$ Cocktail probes for detection of multiple HPV types are indicated by a slash between types. 
$51 \pm 15$ years for HPV-positive patients, and an average age of $61 \pm 14$ years for HPV-negative patients.

Conventional squamous cell carcinoma, not otherwise specified, has been shown to contain detectable HPV in up to $39 \%$ of cases in previous work. ${ }^{22}$ In this study, the HPV-positive rate for squamous cell carcinoma, not otherwise specified, cases was substantially elevated (62\%; 44 of 71 cases) relative to previous reports. One possible explanation for this higher than expected HPV-positive rate is the fact that not all slides were available for review from every case in the current study; therefore, some tumors classified as squamous cell carcinoma, not otherwise specified, may have contained small foci of warty carcinoma or basaloid carcinoma which could potentially skew the results. Squamous cell carcinoma, not otherwise specified, with admixed histologic features of warty carcinoma and/or basaloid carcinoma was previously noted in some invasive squamous vulvar carcinoma cases by Toki et $a l^{18}$ and Kurman et $a l^{32}$ It is also possible that stringent application of morphologic criteria to define basaloid carcinoma and warty carcinoma variants in the current study may have led to an over-representation of squamous cell carcinoma, not otherwise specified. However, another study failed to show a strong relationship between tumor subtype and HPV infection. ${ }^{24}$ Additionally, Ansink et $a l^{41}$ identified lichen sclerosus adjacent to about $37 \%$ of HPV-positive invasive squamous vulvar carcinomas in older women, and Madeleine et $a l^{7}$ reported an increased risk for invasive squamous vulvar carcinoma among women greater than 60 years of age when associated with HPV16 seropositivity and having more than two sexual partners. Perhaps HPV-associated invasive squamous vulvar carcinoma is increasing among the elderly, as it apparently is in younger women, ${ }^{2-4}$ and is not as limited by histologic pattern as previously thought.

Attempts to attach prognostic significance to HPVpositive status in invasive squamous vulvar carcinoma have met with mixed results. Pinto et $a l^{19}$ reported that 5-year patient survival was not significantly different in HPV-positive invasive squamous vulvar carcinoma than in HPV-negative invasive squamous vulvar carcinoma, and that no significant association could be identified between tumor HPV status and prognosis. These findings are in agreement with the work of others. ${ }^{13,30,31}$ However, Kagie et $a l^{24}$ noted that patients with HPVpositive vulvar squamous carcinoma often present at a lower clinical stage, which could improve prognosis. Also, Monk et $a l^{22}$ used life-table analysis to determine that the absence of HPV DNA in invasive squamous vulvar carcinoma and the presence of nodal metastasis were predictive of tumor recurrence and death from vulvar cancer, and Ansink et $a l^{46}$ reported that patients with HPV-positive invasive squamous vulvar carcinoma had a better prognosis than those with HPV-negative tumors.
In contrast, one report ${ }^{31}$ indicated that women with basaloid carcinoma, a tumor type strongly associated with HPV infection in some studies ${ }^{18}$ were 3.5 times more likely to die from vulvar cancer than those with squamous cell carcinoma, not otherwise specified. In the current study, the available clinical data were not always complete, which severely limited any prognostic associations with tumor type or HPV status. However, the presence of nodal metastasis, which has been shown to significantly decrease 5-year survival in patients with invasive squamous vulvar carcinoma ${ }^{5,6}$ was reliably documented in pathology reports reviewed for our study. Our results indicate that invasive squamous vulvar carcinoma cases associated with any HPV type, any high-risk HPV, or HPV16 alone, were significantly less likely to metastasize than HPV-negative tumors. Correcting for possible confounding variables, such as patient age and tumor histology, logistic regression analysis confirmed this association (high-risk HPV odds ratio $0.28,95 \%$ confidence interval 0.09-0.89). Whether these observations reflect a less biologically aggressive tumor type when associated with HPV infection, or are related to clinical issues that we cannot approach with these data, such as earlier disease presentation or an evolving surgical approach with less aggressive, more individualized node sampling, is not clear. Other data available for our study, such as measured tumor size and invasive tumor depth, which may have some prognostic significance, did not correlate with metastatic disease or HPV status. However, the current series revealed that warty carcinoma tended to have a thinner invasive tumor component than squamous cell carcinoma, not otherwise specified (Table 3) which is in contrast to the observations of others. ${ }^{32}$

HPV DNA was detected in regional lymph node metastatic tumor deposits in 9 of 11 cases (82\%) of HPV-positive invasive squamous vulvar carcinoma. This positive rate is higher than reported by Pinto et $a l,{ }^{19}$ who identified HPV DNA in only $23 \%$ of similar cases using L1 consensus HPV primers sets. In the current study, the HPV genotypes detected in nodes were identical to those present in the primary tumors in all but one case, which is consistent with previous reports. ${ }^{19,31}$ In the single discrepant case, one HPV type (HPV33) was identical in both primary tumor and node, while a second type (HPV16) was only present in the metastatic tumor. This high rate of correlating genotypes between primary tumor and metastases demonstrates the reliable performance of the Linear Array HPV Test using paraffin-embedded tissues in our laboratory. Of the nine HPV-positive metastatic tumor deposits in regional lymph nodes, five were warty carcinoma and one was basaloid carcinoma. In previous work, it was noted that warty carcinoma lesions rarely metastasized, although only seven cases were examined in the study. ${ }^{32}$ The significance of this apparent discordance between the prior study and our data is uncertain. 
A limitation of this and similar studies that have investigated the prevalence of HPV types associated with invasive squamous vulvar cancer is that small sample sizes constrain the statistical power to detect small differences between test groups. This same limitation also affects the precision of statistically significant findings as evidenced by the broad confidence intervals surrounding some of our risk estimates. However, the overall sample size in our study is substantially larger than that of most previous investigations of HPV genotypes associated with invasive squamous vulvar cancer and likely suffers less from such limitations. Nevertheless, all results and conclusions of our study should be tempered with an awareness of the contribution that statistical power, precision and effect that sample size must play in interpreting the results.

In conclusion, we applied a sensitive, PCR-based method that detects 37 anogenital HPV genotypes to archived surgical pathology materials, and our results confirm a high prevalence of HPV16 in invasive squamous vulvar carcinoma. HPV 33 was the next most common genotype but other high-risk HPV types were fairly uncommon. Low-risk HPV types were rarely identified as the sole HPV present in invasive squamous vulvar carcinoma, but were occasionally noted in combination with high-risk HPV. While warty carcinoma and basaloid carcinoma histologic patterns correlated with positive HPV status, a surprisingly high percentage of conventional squamous cell carcinoma cases was also positive for HPV, a finding that contrasts with previous studies. Finally, any HPV-positive invasive squamous vulvar carcinoma appeared less likely to metastasize than an HPV-negative tumor, suggesting that HPV status in invasive squamous vulvar carcinoma may have some impact on prognosis. More work is needed to clarify further the relationship between HPV infection and the development and biologic behavior of invasive squamous cell carcinoma of the vulva.

\section{Acknowledgement}

We thank Ms Linda Freeman (OUMC) for her help in retrieving archived paraffin blocks and reports.

\section{References}

1 American Cancer Society. Cancer Facts \& Figures 2007. American Cancer Society: Atlanta, 2007.

2 Al-Ghamdi A, Freedman D, Miller D, et al. Vulvar squamous cell carcinoma in young women: a clinicopathologic study of 21 cases. Gynecol Oncol 2002; 84:94-101.

3 Losch A, Joura EA. Vulvar neoplasia in young women. Gynecol Oncol 1999;75:519-522.

4 Jones RW, Baranyai J, Stables S. Trends in squamous cell carcinoma of the vulva: the influence of vulvar intraepithelial neoplasia. Obstet Gynecol 1997;90: 448-452.

5 National Cancer Institute.US National Institutes of Health website, http://www.cancer.gov.

6 Homesley HD, Bundy BN, Sedlis A, et al. Assessment of current International Federation of Gynecology and Obstetrics staging of vulvar carcinoma relative to prognostic factors for survival (a Gynecologic Oncology Group Study). Am J Obstet Gynecol 1991;164: 997-1003 discussion 1003-1004.

7 Madeleine MM, Daling JR, Carter JJ, et al. Cofactors with human papillomavirus in a population-based study of vulvar cancer. J Natl Cancer Inst 1997;89: 1516-1523 [published erratum appears in J Natl Cancer Inst 1997;89:1516-1523].

8 Khan MJ, Castle PE, Lorincz AT, et al. The elevated 10 -year risk of cervical precancer and cancer in women with human papillomavirus (HPV) type 16 or 18 and the possible utility of type-specific HPV testing in clinical practice. J Natl Cancer Inst 2005;97:1072-1079.

9 Schiffman $\mathrm{M}$, Herrero $\mathrm{R}$, Desalle $\mathrm{R}$, et al. The carcinogenicity of human papillomavirus types reflects viral evolution. Virol 2005;337:76-84.

10 Munoz N, Bosch FX, de Sanjose S, et al. Epidemiologic classification of human papillomavirus types associated with cervical cancer. N Engl J Med 2003;348: 518-527.

11 Clifford GM, Smith JS, Plummer M, et al. Human papillomavirus types in invasive cervical cancer worldwide: a meta-analysis. Br J Cancer 2003;88: $63-73$.

12 van der Avoort IAM, Shirango H, Hoevenaars BM, et al. Vulvar squamous cell carcinoma is a multifactorial disease following two separate and independent pathways. Int J Gynecol Pathol 2005;25:22-29.

13 Andersen WA, Franquemont DW, Williams F, et al. Vulvar squamous cell carcinoma and papillomaviruses: two separate entities? Am J Obstet Gynecol 1991;165:329-336.

14 Costa S, Syrjänen S, Vendra C, et al. Human papillomavirus infections in vulvar precancerous lesions and cancer. J Reprod Med 1995;40:291-298.

15 Engelman DE, Andrade LA, Vassallo J. Human papillomavirus infection and p53 protein expression in vulvar intraepithelial neoplasia and invasive squamous cell carcinoma. Braz J Med Biol Res 2003;36: 1159-1165.

16 Brandenberger AW, Rüdlinger R, Hänggi W, et al. Detection of human papillomavirus in vulvar carcinoma. A study by in situ hybridization. Arch Gynecol Obstet 1992;252:31-35.

17 Park JS, Kurman RJ, Kessis TD, et al. Comparison of preoxidase-labeled DNA probes with radioactive RNA probes for detection of human papillomaviruses by in situ hybridization in paraffin sections. Modern Pathol 1991;4:81-85.

18 Toki T, Kurman RJ, Park JS, et al. Probable nonpapillomavirus etiology of squamous cell carcinoma of the vulva in older women: a clinicopathologic study using in situ hybridization and polymerase chain reaction. Int J Gynecol Pathol 1991;10:107-125.

19 Pinto AP, Schlecht NF, Pintos J, et al. Prognostic significance of lymph node variables and human papillomavirus DNA in invasive vulvar carcinoma. Gynecol Oncol 2004;92:856-865.

20 Rusk D, Sutton GP, Look FY, et al. Analysis of invasive squamous cell carcinoma of the vulva and vulvar 
intraepithelial neoplasia for the presence of human papillomavirus DNA. Obstet Gynecol 1991;77: 918-922.

21 Sutton GP, Stehman FB, Ehrlich CE, et al. Human papillomavirus deoxyribonucleic acid in lesions of the female genital tract: evidence for type 6/11 in squamous carcinoma of the vulva. Obstet Gynecol 1987;70:564-586.

22 Monk BJ, Burger RA, Lin F, et al. Prognostic significance of human papillomavirus DNA in vulvar carcinoma. Obstet Gynecol 1995;85:709-715.

23 Rufforny I, Wilkinson EJ, Lui C, et al. Human papillomavirus infection and p16 ${ }^{I N K 4 a}$ protein expression in vulvar intraepithelial neoplasia and invasive squamous cell carcinoma. J Lower Genital Tract Dis 2005;9:108-113.

24 Kagie MJ, Kenter GG, Zomerdijk-Nooijen Y, et al. Human papillomavirus infection in squamous cell carcinoma of the vulva, in various synchronous epithelial changes and in normal vulvar skin. Gynecol Oncol 1997;67:178-183.

25 Trimble CL, Hildesheim A, Brinton LA, et al. Heterogeneous etiology of squamous carcinoma of the vulva. Obstet Gynecol 1996;87:59-64.

26 Ngan HYS, Cheung ANY, Liu SS, et al. Abnormal expression or mutation of TP53 and HPV in vulvar cancer. Eur J Cancer 1999;35:481-484

27 Hampl M, Sarajuuri H, Wentzensen N, et al. Effect of human papillomavirus vaccines on vulvar, vaginal, and anal intraepithelial lesions and vulvar cancer. Obstet Gynecol 2006;108:1361-1368.

28 Ogunbiyi OA, Sholefield JH, Robertson G, et al. Anal human papillomavirus infection and squamous neoplasia in patients with invasive vulvar cancer. Obstet Gynecol 1994;83:212-216.

29 Iwasawa A, Nieminen P, Lehtinen $\mathrm{M}$, et al. Human papillomavirus in squamous cell carcinoma of the vulva by polymerase chain reaction. Obstet Gynecol 1997;89:81-84.

30 Bloss JD, Liao S-Y, Wilczynski SP, et al. Clinical and histologic features of vulvar carcinomas analyzed for human papillomavirus status: evidence that squamous cell carcinoma of the vulva has more than one etiology. Hum Pathol 1991;22:711-718.

31 Pinto AP, Signorello LB, Crum CP, et al. Squamous cell carcinoma of the vulva in Brazil: prognostic importance of host and viral variables. Gynecol Oncol 1999;74:61-67.

32 Kurman RJ, Toki T, Schiffman MH. Basaloid and warty carcinomas of the vulva. Distinctive types of squamous cell carcinoma frequently associated with human papillomaviruses. Am J Surg Pathol 1993;17:133-145.

33 Sagerman PM, Choi YJ, Hu Y, et al. Human papilloma virus, vulvar dystrophy, and vulvar carcinoma: differential expression of human papillomavirus and vulvar dystrophy in the presence and absence of squamous cell carcinoma of the vulva. Gynecol Oncol 1996;61: 328-332.

34 Hørding U, Kringsholm B, Andreasson B, et al. Human papillomavirus in vulvar squamous-cell carcinoma and in normal vulvar tissues: a search for a possible impact of HPV on vulvar cancer prognosis. Int J Cancer 1993;55:394-396.

35 Hørding U, Junge J, Daugaard S, et al. Vulvar squamous cell carcinoma and papillomaviruses: indications for two different etiologies. Gynecol Oncol 1994;52: 241-246.

36 Venuti A, Marcante ML. Presence of human papillomavirus type 18 DNA in vulvar carcinomas and its integration into the cell genome. J Gen Virol 1989;70:1587-1592.

37 Srodon M, Stoler MH, Baber GB, et al. The distribution of low- and high-risk HPV types in vulvar and vaginal intraepithelial neoplasia (VIN and VaIN). Am J Surg Pathol 2006;30:1513-1518.

38 Dunn ST, Allen RA, Wang S, et al. DNA extraction: an understudied and important aspect of HPV genotyping using PCR-based methods. J Virol Methods 2007;143: 45-54.

39 Woo YL, Damay I, Stanley M, et al. The use of HPV Linear Array Assay for multiple HPV typing on archival frozen tissue and DNA specimens. J Virol Meth 2007;142:226-230.

40 Nuovo GJ, Delvenne P, MacConnell P, et al. Correlation of histology and detection of human papillomavirus DNA in vulvar cancers. Gynecol Oncol 1991;43: 275-280.

41 Ansink AC, Krul MRL, De Weger RA, et al. Human papillomavirus, lichen sclerosus, and squamous cell carcinoma of the vulva: detection and prognostic significance. Gynecol Oncol 1994;52:180-184.

42 Hampl M, Ramp R, Bender HG, et al. A case of a pT3, HPV 52-positive vulvar carcinoma in an 18-year-old woman. Gynecol Oncol 2006;101:530-533.

43 Hørding U, Daugaard S, Iversen AKN, et al. Human papillomavirus type 16 in vulvar carcinoma, vulvar intraepithelial neoplasia, and associated cervical neoplasia. Gynecol Oncol 1991;42:22-26.

44 Husseinzadeh N, DeEulis T, Newman N, et al. HPV changes and their significance in patients with invasive squamous cell carcinoma of the vulva: a clinicopathologic study. Gynecol Oncol 1991;43: 237-241.

45 Yang B, Hart WR. Vulvar intraepithelial noeplasia of the simplex (differentiated) type. A clinicopathologic study including analysis of HPV and p53 expression. Am J Surg Patholol 2000;24:429-441.

46 Riethdorf S, Neffen EF, Cviko A, et al. p16 $6^{\text {ink } 4 A}$ expression as biomarker for HPV16-related vulvar neoplasias. Hum Pathol 2004;35:1477-1483. 